\title{
Exact Command Tracking Control Computations without Integration
}

\author{
TAIN-SOU TSAY \\ Department of Department of Aeronautical Engineering \\ National Formosa University \\ 64, Wen-Hua Road, Huwei, Yunlin, TAIWAN
}

\begin{abstract}
A digital controller for exact command tracking control without integration is derived from a periodic series. The ratios of adjacent values will be converged to unities after the output has tracked the reference input command. Integration in control loop usually introduces phase lag to slow command response and degrade performance.
\end{abstract}

Key-Words: - Exact command Tracking, digital control, Zero Steady-State Error

Received: July 9, 2021. Revised: November 6, 2021. Accepted: November 20, 2021. Published: January 3, 2022.

\section{Introduction}

For discrete-time unit feedback control systems, the control sequences $G\left(j T_{S}\right)$ are usually functions of the difference between the sampled reference input and output of the plant [1-3]. $T_{S}$ is the sampling interval. They are linear control sequences. In this literature, ratios of $G\left((k+1) T_{S}\right)$ to $G\left(k T_{S}\right)$ of the control sequences will be formulated as a nonlinear function of the reference input command and the output of the plant. The value of $G\left(k T_{S}\right)$ is the control effort of the plant at time interval between $(k-1) T_{S}$ and $k T_{S}$. Thus, the considered system is closed with $G\left(j T_{S}\right)$. The output of the plant will track the reference input exactly after $G\left((k+1) T_{S}\right) / G\left(k T_{S}\right)$ converged to be unities. It implies that $G\left(k T_{S}\right)$ will be converged to a steadystate value for a constant reference input applied. The stability of the closed-loop system is guaranteed by selecting the proper function of ratios $G\left((k+1) T_{S}\right) / G\left(k T_{S}\right)$. It will be proven that the considered system with the proposed $G\left(k T_{S}\right)$ is a stable negative feedback control system.

\section{Propose Method}

A series with time period $T_{S}[1-3]$ can be written as in the form of

$$
G\left(j T_{S}\right), j=1,2,3, . ., n, n+1, \ldots .
$$

where $G\left(k T_{S}\right)$ represents a constant value between time interval between $(j-1) T_{S}$ and $j T_{S}$. For simplicity, the representation of $G\left(k T_{S}\right)$ will be replaced by $G(j)$ in following evaluations. The ratios $G(j+1) / G(j)$ of the series are defined as

$$
F(j) \equiv G(j+1) / G(j), j=1,2,3, \ldots, n, n+1, \ldots . .
$$

Eq.(2) gives the value of $G(n+1)$ approaches to be a constant value when the value of $F(n)$ approaches to be unity. Now, the problem for closing the considered system with exact command tracking is to find the formula of $F(j)$ which is the function of reference input command and output of the plant. $G(n+1)$ will be used as the input of the considered system. Considering a possible series to close the considered system, it is

$$
G(n+1)=\left\lfloor\sum_{i=0}^{m} a_{i}\left(R(n) / Y_{S}(n)\right)^{i}\right\rfloor G(n) ;
$$

where $R(n)$ is the reference input command and $Y_{S}(n)$ is the non-zero sampled output of the plant $\mathrm{Y}$ at the sampling interval $n T_{S}$. Assume that the reference input command has been tracked by the control effort $G(j)$, Eq.(3) becomes

$$
G(n+1)=\sum_{i=0}^{m} a_{i} G(n)
$$

For steady-state condition, $G(n+1)$ approaches to be a constant value. It gives $\sum_{i=0}^{m} a_{i}=1$.

Rearranging the Eq. (3) and taking the derivative of it with respect to $Y_{S}(n) / R(n)$, we have 


$$
F(n)=\sum_{i=0}^{m} a_{i}\left(Y_{S}(n) / R(n)\right)^{-i}
$$

and

$$
\partial F(n) / \partial\left(Y_{S}(n) / R(n)\right)=-\sum_{i=0}^{m} i a_{i}\left(Y_{S}(n) / R(n)\right)^{-i-1}
$$

The sufficient but not necessary condition for Eq.(6) less than zero is $a_{i}>0$ for $Y_{S}(n) / R(n) \cong 1$ and Eq.(5) is rewritten as in the form of

$$
F(n)=\sum_{i=0}^{m} a_{i}\left\|Y_{S}(n) / R(n)\right\|^{-i}
$$

$a_{i}>0$ will be used in following evaluations. Negative value of Eq.(6) represents the closed-loop system with Eq.(3) activated as a negative feedback system around the equilibrium condition; i.e., $Y_{S}(n)=R(n)$. These statements will be illustrated by the first order polynomial:

$$
G(n+1)=\left[(1-\beta) R(n) / Y_{S}(n)+\beta\right] G(n)
$$

where $\beta$ satisfies constrains stated above and becomes a adjustable parameter. The ratios $F(n)$ becomes

$$
F(n)=(1-\beta) /\left(Y_{S}(n) / R(n)\right)+\beta
$$

Taking the derivative of Eq. (9) with respect to $Y_{S}(n) / R(n)$, we have

$$
\partial F(n) / \partial\left(Y_{S}(n) / R(n)\right)=-(1-\beta) /\left(Y_{S}(n) / R(n)\right)^{2}
$$

For negative value of Eq.(10), the value of $\beta$ must be less than one. The suitability of the proposed nonlinear digital controller is based upon this characteristic. Fig. 1 shows ratios $Y_{S}(n) / R(n)$ versus $F(n)=G(n+1) / G(n)$ represented by Eq.(8) for $\beta$ $=0.9,0.7,0.5$ and 0.3 ; respectively. Fig. 1 shows that the value of $F(n)$ is less than one for $Y_{S}(n)$ greater than $R(n)$, then the value of $G(n+1)$ will be decreased; and the value of $F(n)$ is greater than one for $Y_{S}(n)$ less than $R(n)$, the value of $G(n+1)$ will be increased. This implies that the controlled system connected with Eq.(8) will be regulated to the equilibrium point $\left(Y_{S}(n) / R(n)=1\right)$ and gives a negative feedback control system for deviation from equilibrium point. One can adjust $\beta$ to get desired regulating characteristic. Certainly, other tracking functions can be formulated and proposed also for the considered system, if its derivative with respect to $Y_{S}(n) / R(n)$ is negative.

Fig. 2 shows the connected system configuration in which Eq.(8) and output of the nonlinear controller are modified for negative control swing. The $\mathrm{C}(\mathrm{z})$ is the digital compensation for better performance. Eq.(8) is rewritten as

$$
G(n+1)=\left[(1-\beta)\left(R+Y_{o}\right) /\left(Y_{S}(n)+Y_{O}\right)+\beta\right] G(n)
$$

where $Y_{o}$ is the desired negative control swing, $Y_{S}(n)$ is the sampled value with hold of the plant output at sampling interval $n T_{S}$, and $U$ is the sampled value with hold of the controller output. The values of $G(n)$ and $F(n)$ will be all positive for the summation of $Y_{S}(n)$ and $Y_{o}$ (or $R$ and $Y_{o}$ ) is greater than zero with a specified value of $Y_{o}$. All positive values will give better continuities, regulating characteristics of the series. Note that singularity of Eq.(8) for $Y_{S}(n)=0$ is avoided by use of Eq.(11). Eq.(11) implies

$$
\begin{gathered}
F(j)=\left[(1-\beta)\left(R+Y_{o}\right) /\left(Y_{S}(j)+Y_{o}\right)+\beta\right], j=1,2,3, . . \\
n, n+1, \ldots .
\end{gathered}
$$

and inputs of the plant $u(n+1)=G(n+1)$ are replaced by

$$
u(n+1)=G(n+1)-Y_{o} / P(0)
$$

for the negative swing control with positive values of $\beta, G(j)$ and $F(j)$.

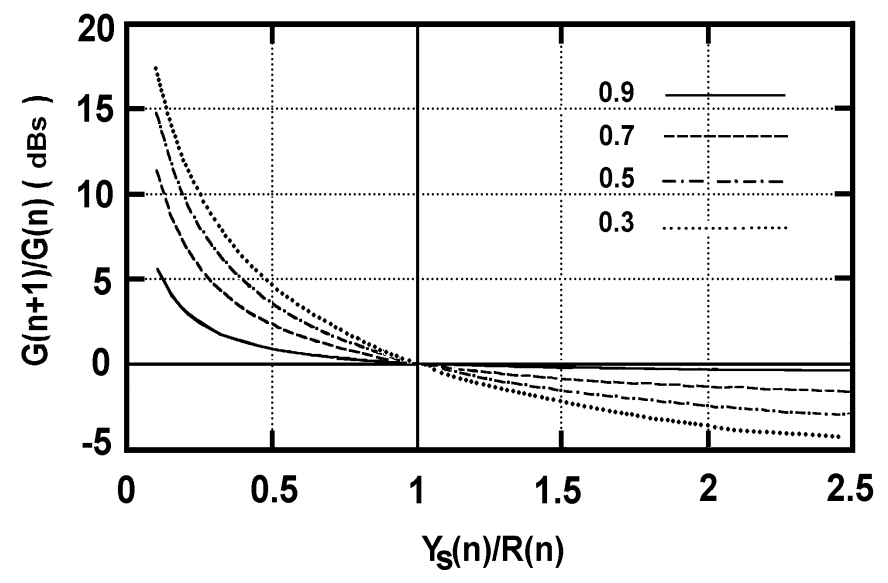

Fig.1. $\mathrm{G}(\mathrm{n}+1) / \mathrm{G}(\mathrm{n})$ Versus $Y_{S}(n) / R(n)$ for $\beta=0.9,0.7,0.5$, and 0.3 . 


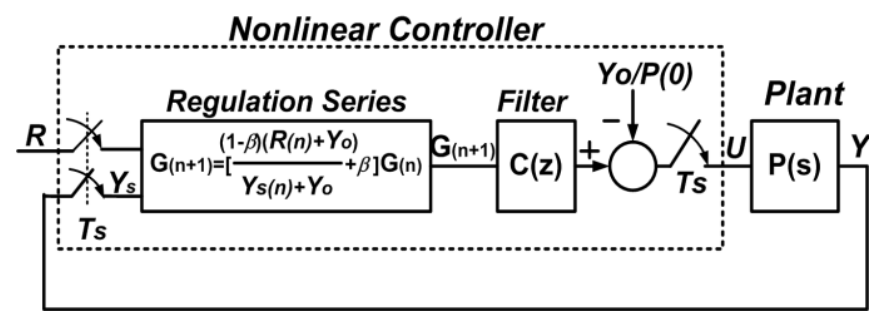

Fig.2. The Nonlinear Digital Controller.

\section{Numerical Examples}

Example 1: The design example [4] is

$$
P_{o}(s)=\frac{100}{s(1+0.1 s)}
$$

After it has been closed with feed-forward gain 0.03 , the DC gain of the closed- loop subsystem $P(s)$ is unity. The sampling period $T_{S}=1 / 40$ second is selected. Time responses of the overall system with the nonlinear digital controller for $\beta=0.95$ is shown in Fig.3. The amplitude of reference inputs $R(j)$ between 0 and 2 seconds are equal to 1 ; between 2 and 6 seconds are equal to -0.2 , between 6 and 9 seconds are equal to 0.6 , and between 9 and 12 seconds are equal to 1.2 , in which gives reference command $R(j)$ (solid-line), plant output $Y_{S}(j)$ (dot-line), control effort $G(j)$ (dash-line), and ratios $F(j)$ (dash-dot-line) of $G(j)$. Fig.3 shows that $G(j)$ and $F(j)$ are all positive while the value of output $Y_{S}(j)$ tracking the negative value of the reference input $R(j)$ exactly. Fig.3 shows also that ratios $F(j)$ are converged to be unities quickly; i.e., the controlled output tracks the reference input quickly and exactly.

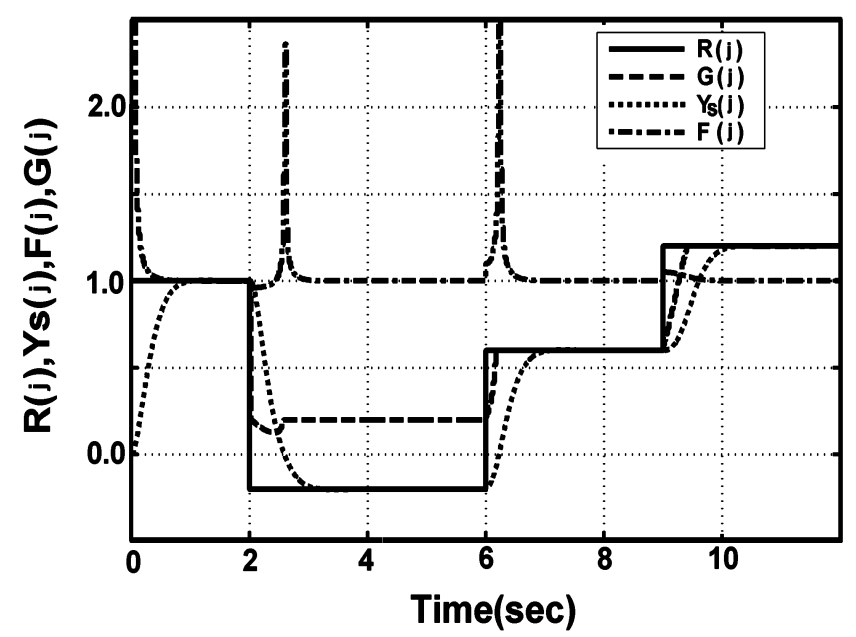

Fig.3. Time Responses of the Design Example for $\beta=0.95$.
Example 2: Consider the very high order plant:

$$
P_{3}(s)=\frac{1}{(s+1)^{20}}
$$

Parameters of the nonlinear controller are $\beta=0.5$ and $T_{S}=25 \mathrm{~ms}$. Fig. 4 shows time response of the controlled system, in which gives reference input $R(n)$ (dash-line), output Y(solid-line), Time series $G(n)$ (dot-line), and ratios $F(n)$ (dash-dot-line) of $G(n)$. It gives good performance and zero steadystate errors. The phase-lead filter $\mathrm{C}(\mathrm{z})$ is in the form of

$$
C(z)=\left.\frac{0.8 s+1}{0.02 s+1}\right|_{s=\frac{2}{T_{S}} z+1}
$$

Fig. 4 shows the considered plant is a large time-lag system. The high order system model is usually used to describe the industry process for replacing pure time-delay(e.g. $e^{-T_{d} s}$ ). Such that conventional analysis and design techniques can be applied[5,6]. Fig. 4 shows the proposed method can be applied to a large time-delayed system.

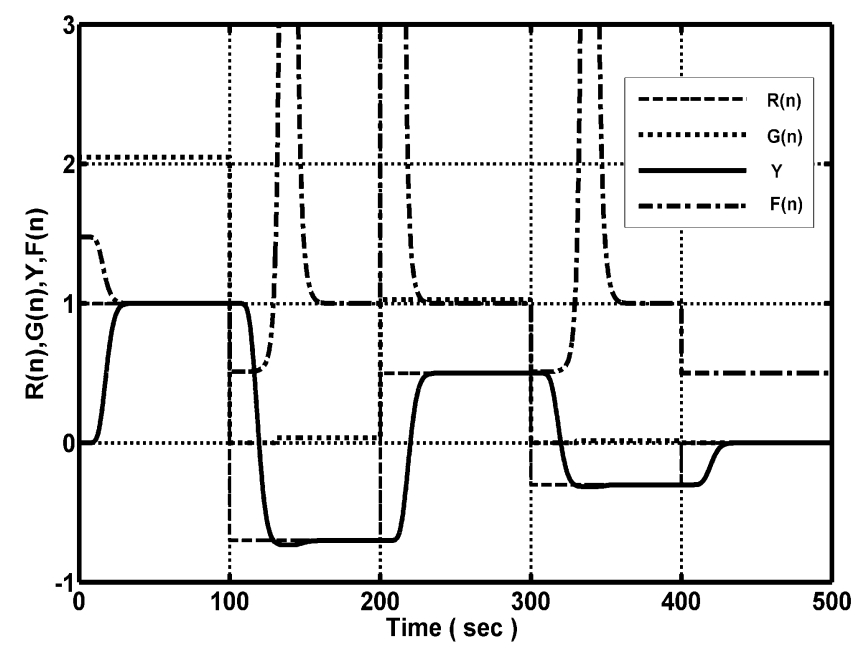

Fig.4. Time responses of Example 2 with $\mathrm{C}(\mathrm{z})$ for $\beta=0.5 ; T_{S}=25 \mathrm{~ms}$.

Final results and four other methods are presented for comparison and show the merit of the proposed method. They are Ziegler-Nichols method[7-9] for finding PI and PID compensators, Zhuang et al. [10] for finding PI compensator and Majhi[11,12] for finding PI compensator. Parameters of four found compensators are given below: 
(1)ZN(PI) : $K_{p}=0.585$ and $K_{i}=0.0305$.

(2)ZN(PID): $K_{p}=0.77256, K_{i}=0.05088$ and $K_{d}=4.9135$.

(3)Majhi's(PI) : $K_{p}=0.5097$ and $K_{i}=0.0443$.

(4)Zhuang's(PI): $K_{p}=0.473$ and $K_{i}=0.058$.

Time responses are shown in Fig.5. Table 1 gives integration of absolute error(IAE) and integration of square error(ISE) of them. From Table 1 and Fig.5, one can see that the proposed method gives better performance than those of other methods.

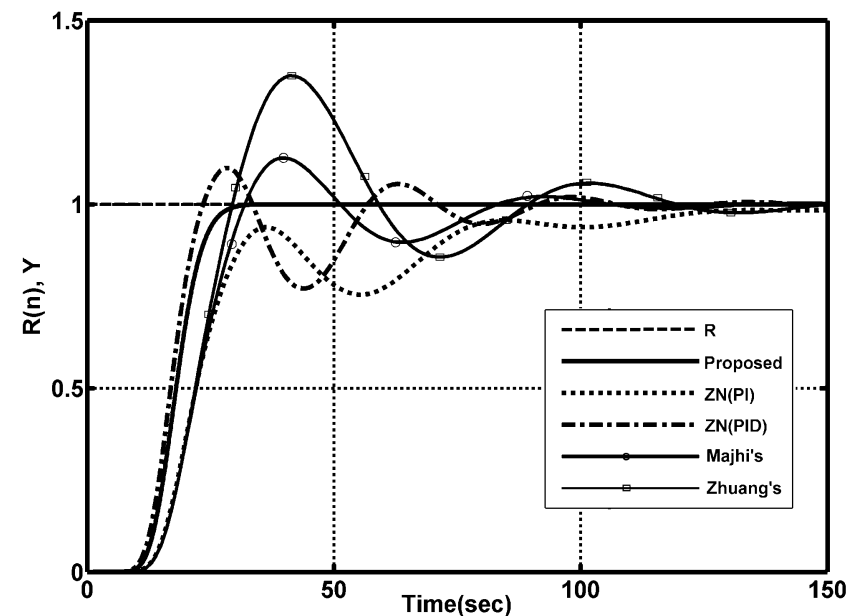

Fig.5. Time Responses of Example 2 with Different Control Methods.

Table 1. IAE and ISE Errors of Example 2 with Different Control Methods.

\begin{tabular}{|c|l|l|l|l|l|}
\hline Methods & Proposed & ZN(PI) & ZN(PID) & Majji & Zhuang \\
\hline IAE & 16.010 & 21.227 & 16.216 & 20.190 & 21.814 \\
\hline ISE & 18.337 & 32.708 & 22.970 & 26.829 & 32.912 \\
\hline
\end{tabular}

Example 3: Consider a gas turbine engine with plant transfer function matrix[13-14].

$P_{3}(s)=\frac{1}{\Delta(s)}\left[\begin{array}{rr}2533+1515.33 s & 1805947+1132094.7 s \\ +14.9 s^{2} & +95150 s^{2} \\ 12268.8+8642.68 s & 2525880+1492588 s \\ +85.2 s^{2} & +124000 s^{2}\end{array}\right]$

where $\Delta(s)=2525+3502.7 s+1357.3 s^{2}+113.22 s^{3}+s^{4}$. It is a $2 \times 2$ multivariable plant. The steady-state gain of open loop $P_{3}(s)$ is in the form of

$$
P_{5}(0)=\left[\begin{array}{cc}
1.00316 & 715.2265 \\
4.85893 & 1000.3485
\end{array}\right]
$$

A pre-compensating matrix $P_{3}^{-1}(0)$ is first applied to decouple the plant in low-frequency band. Then, two digital filters are used in the diagonal to filter outputs of two time series for speeding up transient responses. They are in the form of

$$
\mathrm{C}_{1}(\mathrm{z})=\left.\frac{0.75 \mathrm{~s}+1}{0.15 \mathrm{~s}+1}\right|_{\mathrm{s}=\frac{2}{\mathrm{~T}_{\mathrm{s}}} \mathrm{z}-1}
$$

and

$$
\mathrm{C}_{2}(\mathrm{z})=\left.\frac{0.60 \mathrm{~s}+1}{0.25 \mathrm{~s}+1}\right|_{\mathrm{s}=\frac{2 \mathrm{z}-1}{\mathrm{~T}_{\mathrm{s}} \mathrm{z}+1}}
$$

where $T_{s}=25 \mathrm{~ms}$ is the sampling period. Fig.6 shows time responses of this controlled system for $\beta=0.5$. It shows that the proposed control scheme can be applied to the multivariable feedback control system also.

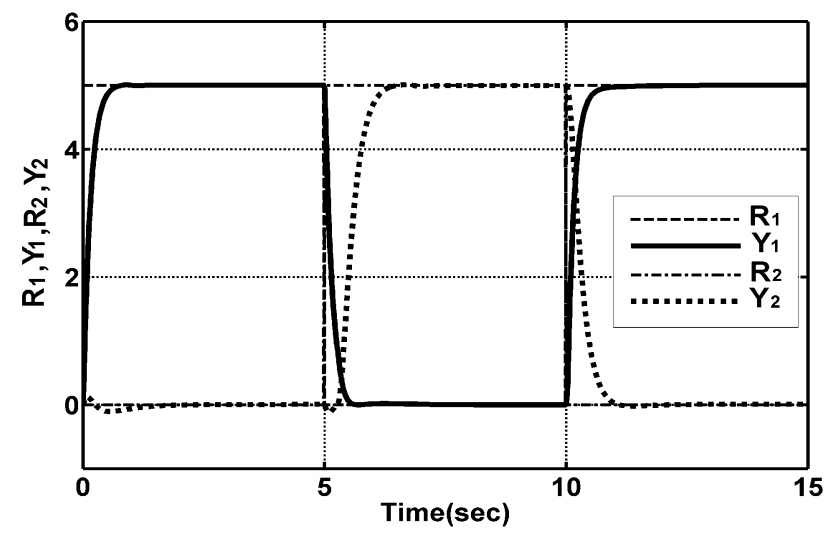

Fig.6. Time Responses of Example 5 for $\beta$ $=0.5$ and $T_{S}=25 \mathrm{~ms}$.

\section{Conclusions}

A new nonlinear digital controller has been proposed for analyses and designs of sampled-data feedback control systems. It gave exact command tracking without integration; i.e., zero steady-state error. The convergence of ratios was illustrated by one servo system example and two complicated examples. From simulation results, it can be seen that the nonlinear digital controller provided another possible control scheme for exact command tracking without integration.

\section{References:}

[1] K. Ogata, Discrete-Time Control System, Prentice-Hall Inc. Englewood Cliffs, NJ, 1994.

[2] G. F. Franklin, et al, Feedback Control Dynamics, 7/e, Pearson Education North Asia, Ltd., Hong Kong, 2015. 
[3] C. L.Phillips, and H. T. Nagle, Digital Control System Analyses and Designs, 4/e, Prentice-Hall Inc., Englewood Cliffs, NJ, 2014.

[4] G. S.Buja, and A. Souliaev, "A Variable Structure Controller," IEEE Trans. on Automatic Control, Vol.33, 1988, pp.206-209.

[5] G. F. Franklin, and J. D Powelll, Feedback Control Dynamics, Addison-Wesley Publishing Company, 1986.

[6] G. F. Franklin, J. D. Powell and M. L. Workman, Digital Control of Dynamics, Ellis-Kagle Press., Half Moon Bay, CA, 2006.

[7] J. G. Ziegler and N. B. Nichols, Optimum Setting for Automatic Controller, Transactions of ASME, Vol.65, 1942, pp.759-768.

[8]K. J. Âström, T. Hägglund, Revisting the Ziegler-Nichols step responses method for PID control, Journal of Process Control, Vol.14, 2004, pp.635-650.

[9]K. J. Áström and C. C. Hang, Towards Intelligent PID Control, Automatica, Vol.28, pp.1-9, 1991.

[10]M. Zhuang and D. P. Atherton, Automatic Tuning of Optimum PID Controllers, IEE Control Theory Application, Vol.140, 1993, pp.216-224.

[11] S. Majhi and D.P. Atherton, Auto-tuning and controller design for process with small time delays, IEE Proc. Control Theory Application, Vol.146, pp.415-425, 1999.

[12]S. Majhi, On-line PI Control of Stable Process, Journal of Process Control, Vol.15, 2005, pp.859-867.

[13] D. Q. Mayne, Sequential Design of Linear Multivariable Systems, Proceeding of IEE, Vol.126, 1979, pp.568-572.

[14]T. S. Tsay, A Sequential Design Method for Multivariable Feedback Control Systems, WSEAS Transactions on Systems, Vol.8, No.12, 2009, pp.1294-1304.

\section{Creative Commons Attribution}

\section{License 4.0 (Attribution 4.0} International , CC BY 4.0)

This article is published under the terms of the Creative Commons Attribution License 4.0 https://creativecommons.org/licenses/by/4.0/deed.en US 